\title{
Retinal vascular occlusion after vitrectomy with retrobulbar anesthesia-observational case series and survey of literature
}

\author{
Christoph Tappeiner • Justus G. Garweg
}

Received: 7 June 2011 /Revised: 3 July 2011 / Accepted: 28 July 2011 / Published online: 18 August 2011

(C) Springer-Verlag 2011

\begin{abstract}
Background Severe postoperative loss of vision has been occasionally reported as a rare complication of retrobulbar anesthesia, and several possible causes have been proposed in the literature. In this work, our own and other investigators' experiences with these complications are surveyed with a view to identifying its pathophysiology. Patients This observational case series refers to six patients who presented during a 3-month period with occlusion of either the central artery itself $(n=3)$ or a branch thereof $(n=$ 3) 2-14 days after uneventful vitreoretinal surgery following retrobulbar anesthesia with a commercial preparation of mepivacaine (1\% Scandicain ${ }^{\circledR}$, Astra Chemicals, Sweden) containing methyl- and propyl parahydroxybenzoate as preservatives.

Results Three of the patients carried risk factors, which were medically controlled. In three individuals, vasoocclusion was observed after a second vitreoretinal intervention, which was performed 3-12 months after uneventful primary surgery. Good visual recovery was observed in only one instance.

Conclusions In patients who were anesthetized with preservative-free mepivacaine, no vasoocclusion occurred. In individuals who were anesthetized with mepivacaine
\end{abstract}

C. Tappeiner

Department of Ophthalmology, Inselspital, University of Bern,

Bern, Switzerland

J. G. Garweg $(\bowtie)$

Clinic for Vitreoretinal Disease, Swiss Eye Institute,

University of Bern,

Bremgartenstrasse 119,

3012 Bern, Switzerland

e-mail: justus.garweg@eye-institute.ch containing the preservatives methyl- and propyl parahydroxybenzoate, a tenfold increase in the incidence of eyes requiring re-operation was documented, with a 2- to 14-day lapse in the onset of vasoocclusion. These findings reveal a possible implication of preservatives contained in the local anesthetic solution for the vasoocclusive events. Due to this potential hazard, the use of preservative-free preparations of local anesthesia in ocular surgery is emphasized in order to prevent this sight-threatening complication.

Keywords Retinal vascular occlusion - Mepivacaine · Adverse event $\cdot$ Retrobulbar anesthesia $\cdot$ Preservatives . Parahydroxybenzoate

\section{Introduction}

In anterior-segment surgery, retrobulbar anesthesia has been largely superseded by topical narcosis. To avoid the necessity for general anesthesia in vitreoretinal surgery, blockage of the retrobulbar nerve using Atkinson's technique is now widely implemented to effect akinesia and analgesia. Complications of this procedure (e.g., hemorrhage, iatrogenic injection of the local anesthetic into the optic-nerve sheath or perforation of the ocular globe) are rare, but may lead to a dramatic and permanent loss of vision. In this observational study, we describe a case series of six patients who developed retinal-artery occlusion after uneventful pars-plana vitrectomy under conditions of retrobulbar anesthesia. On the basis of our own and other investigators' experiences with this complication, we discuss its possible etiology, including an anestheticinduced vasospasm and the neurotoxicity of preservative agents. 


\section{Case series}

During a 3-month period, six patients presented with acute visual disturbance 2-14 days after uneventful vitrectomy: with air for the treatment of either an epiretinal membrane $(n=$ $1)$ or vitreous hemorrhaging $(n=1)$; with a $15 \% \mathrm{SF}_{6}$ gas tamponade for a macular hole $(n=1)$; and with air after the removal of silicone oil in conjunction with successful retinal detachment surgery for either PVR $(n=2)$ or a persistent macular hole $(n=1)$. Blockage of the retrobulbar nerve using Atkinson's technique was achieved by injecting $3.5 \mathrm{ml}$ of a commercial preparation of mepivacaine (1\% Scandicain ${ }^{\circledR}$, Astra Chemicals, Sweden), which contained methyl- and propyl parahydroxybenzoate as preservatives. The drug solution (which contained no adrenalin) was injected 30 min prior to the onset of surgery in each case. Uneventful vitreoretinal surgery (involving a 23-gauge needle microincision) was completed within 30-60 min without intraoperative fluctuations in intraocular pressure (IOP). On the 1 st postoperative day, none of the operated eyes evinced signs of irregularities in retinal perfusion, and the IOP followed a normal course in each case. No functional abnormalities were reported. Between the 2nd and the 14th postoperative days, each of the patients experienced a central loss of vision, which was not related to the presence of either residual air or $\mathrm{SF}_{6}$-gas. Examination revealed a normal IOP (11-19 $\mathrm{mm}$ of $\mathrm{Hg}$ ) in all instances. The anterior segment was quiet, except for mild conjunctival hyperemia and, in two cases, subconjunctival hemorrhaging at the site of sclerotomy. Retinal biomicroscopy revealed hemorrhagic ischemic retinopathy and vascular occlusion in all instances.

The decrease in visual acuity was subsequently revealed to be due to occlusion of either the central retinal artery $(n=3)$ or a branch thereof $(n=3)$ (Figs. 1 and 2). As postoperatively

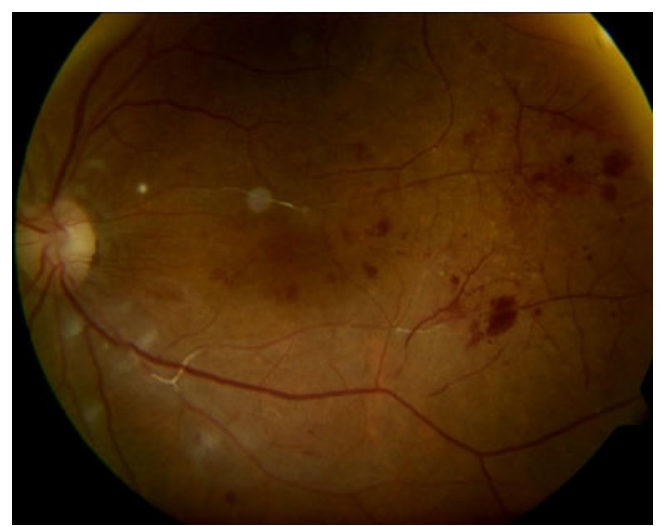

Fig. 1 Retinal image of a 74-year-old female patient (see Table 1) who had undergone re-vitrectomy with retrobulbar anesthesia for a persistent macular hole. Five days after the removal of the silicon oil and its replacement with air, occlusion of the retinal artery was revealed. The best visual acuity prior to the removal of the silicone oil was 20/50; the final visual acuity ( 1 year after surgery) was 20/200

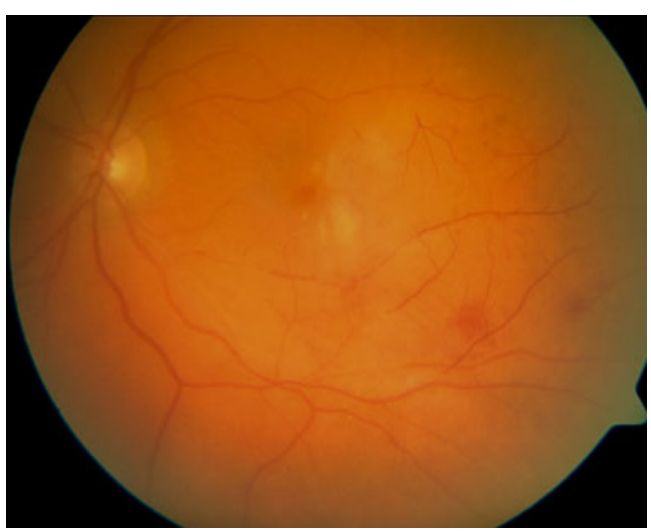

Fig. 2 Retinal image of a 66-year-old female patient (see Table 1) who had undergone uncomplicated vitrectomy with retrobulbar anesthesia for a macular hole using $15 \% \mathrm{SF}_{6-}$ gas filling. Occlusion of the retinal artery occurred 2 days after surgery

assessed by carotid Doppler sonography, echocardiography, transesophageal echography, and 24-h electrocardiography, no critical systemic risk factors for vasoocclusive events were identifiable in any of the subjects. Nevertheless, three of the patients had a history of treated arterial hypertension; one of these suffered from diabetes mellitus, and one had undergone prophylactic treatment with aspirin for cardiac problems after surgery for an aortic aneurysm 23 years earlier (Table 1). Beyond confirmation of an optimal therapy of their preexisting underlying diseases, all patients received (after exclusion of contraindications) a local IOP lowering therapy with timolol $0.5 \%$ for 10 days, aspirin $100 \mathrm{mg} /$ day for 3 months, and a retard preparation of nifedipine $20 \mathrm{mg} /$ day for 1 month. One year after surgery, visual acuity was poor in five of the patients (20/200 in four individuals; light perception in one) and excellent in one (20/20).

\section{Discussion}

Occlusion of the retinal artery after intraocular surgery with anesthesia of the retrobulbar nerve has been reported in small cohorts of patients during the past few decades (Table 1). Although no direct explanation for the vasoocclusive event was furnished in the pertinent publications, various causative factors were discussed, including iatrogenic injuring of the optic nerve during the injection of the local anesthetic and traumatic retrobulbar hemorrhage with an ensuing increase in IOP or a compression of the ocular globe [1-4]. Hørven et al. demonstrated that the anesthetic solution itself could cause a decrease in blood flow within the ophthalmic artery by exerting a vasoconstrictive effect [5]. Klein et al. suggested that in susceptible individuals, the injection process per se could induce traumatic spasms 


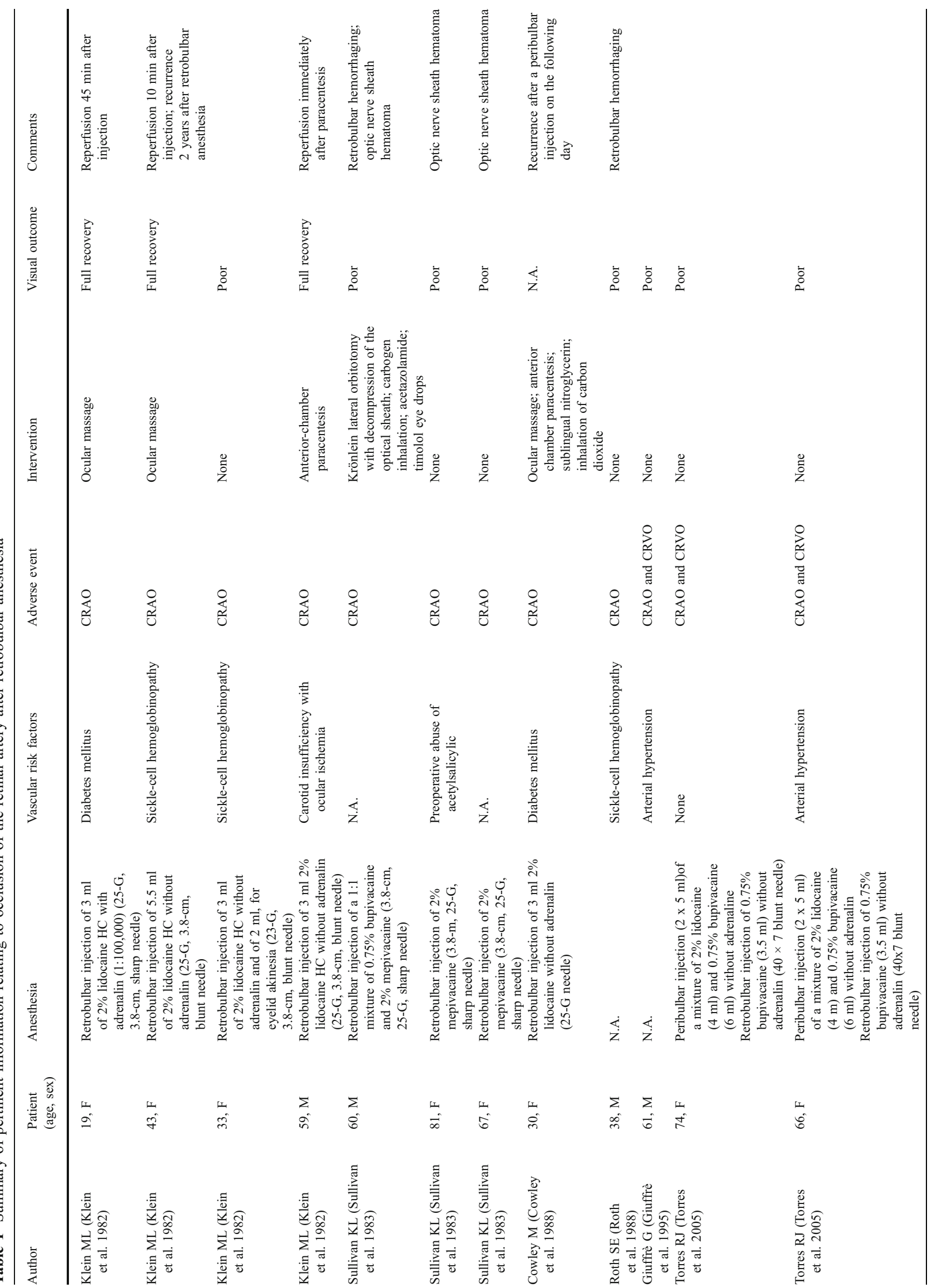




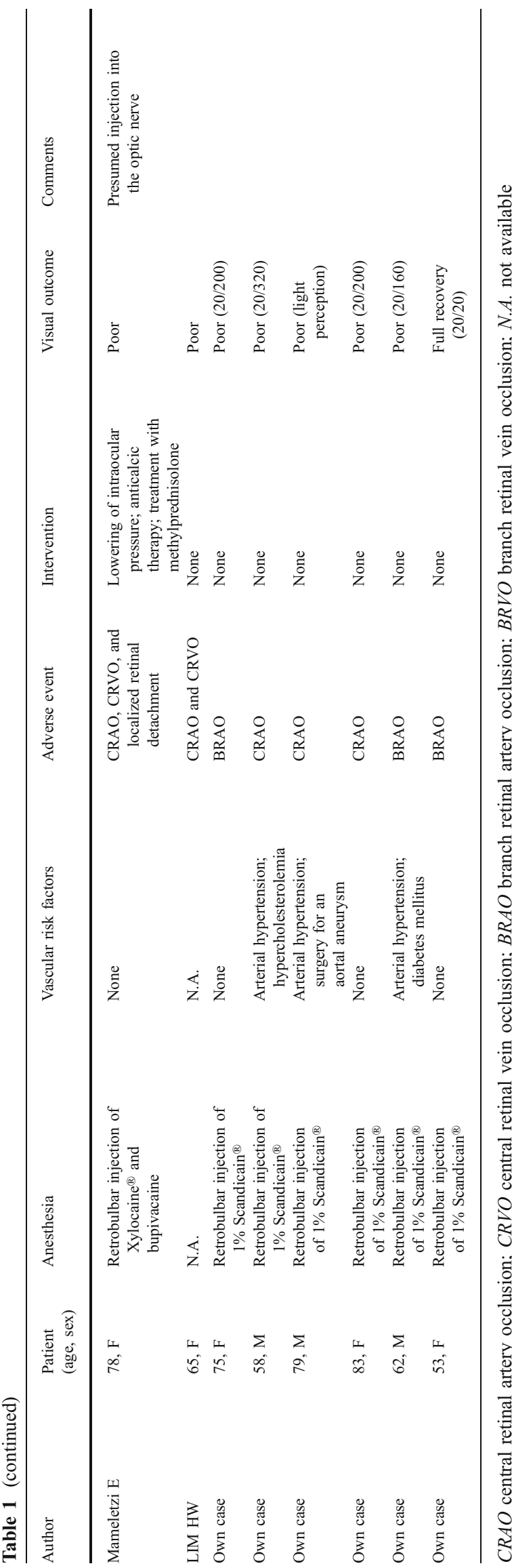

of the central retinal artery [3], in analogy to the finding that stimulation of the retinal arterioles in cats can trigger a vasospasm [6].

During the 3-month time-span of the published findings, 141 patients have undergone vitrectomy in the same context at our own center, the incidence of postoperative vasoocclusive events among these individuals being $4.3 \%$ (six patients). After the exclusion of obvious patientrelated factors and of irregularities in surgical devices and machines including intra- and early postoperative IOP and infusion flow, the commercial preparation of mepivacaine $\left(1 \%\right.$ Scandicain $\left.{ }^{\circledR}\right)$ was identified as a possible cause of the vasoocclusive event. This local anesthetic contains methyl- and propyl parahydroxybenzoate as preservatives. The same local anesthetic, but in the absence of preservatives, had been consistently used by the same surgeon (specialized senior ophthalmologist) without encountering a single instance of the complication in a total of almost 900 vitrectomies. No signs for inadvertent intrathecal injection of the anesthetic could be found in our six patients.

Astro Chemicals informed us that vascular occlusion and neurotoxicity had been reported after an intrathecal application of Scandicain ${ }^{\circledR}$. Saiki et al. have discussed the neurotoxic effects of the preservatives methyl- and propyl parahydroxybenzoate, having observed cases of paraplegia after the intrathecal application of chemotherapeutic drug solutions containing these agents [7]. Previously, Nathan and Sears had demonstrated that the topical application of a $0.1 \%$ solution of methyl hydroxybenzoate to the roots of the spinal nerve induced a conduction blocking [8]. On the basis of these disturbing indications, we filed a report to the Swiss Health Authorities. In parallel to this action, Astro Chemicals was compelled to include among the contraindications of Scandicain ${ }^{\circledR}$ a potential risk of vasoocclusion in a retrobulbar application.

Interestingly, three of our six patients had undergone retrobulbar anesthesia with Scandicain ${ }^{\circledR}$ 3-12 months before the initial vitreoretinal intervention without evincing clinically detectable signs of vascular occlusion. Therefore, a long-lasting or even persistent toxic effect resulting in cumulative toxicity can not be excluded.

Although we cannot categorically state that a correlation exists between the presence of methyl- and propyl parahydroxybenzoate in solutions of mepivacaine and delayed vascular occlusion after retrobulbar anesthesia, our findings may suggest such an association. The 2- to 14-day timelapse between the induction of anesthesia and the onset of vascular occlusion is indicative of a toxic effect on the vascular endothelium rather than of a vasospastic event that might be triggered by undetected intra- and early postoperative rises in IOP, which would be expected to occur immediately. 
In conclusion, the existing findings indicate a possible vasculotoxic effect of preservatives included in commercial preparations of mepivacaine. We thus strongly recommend the use of preservative-free solutions of this local anesthetic in ocular surgery.

Financial disclosure None of the authors has either a financial or a proprietary interest in any of the presented materials or methods.

\section{References}

1. Sullivan KL, Brown GC, Forman AR, Sergott RC, Flanagan JC (1983) Retrobulbar anesthesia and retinal vascular obstruction. Ophthalmology 90:373-377
2. Roth SE, Magargal LE, Kimmel AS, Augsburger JJ, Morrison DL (1988) Central retinal-artery occlusion in proliferative sickle-cell retinopathy after retrobulbar injection. Ann Ophthalmol 20:221224

3. Klein ML, Jampol LM, Condon PI, Rice TA, Serjeant GR (1982) Central retinal artery occlusion without retrobulbar hemorrhage after retrobulbar anesthesia. Am J Ophthalmol 93:573-577

4. Cowley M, Campochiaro PA, Newman SA, Fogle JA (1988) Retinal vascular occlusion without retrobulbar or optic nerve sheath hemorrhage after retrobulbar injection of lidocaine. Ophthalmic Surg 19:859-861

5. Hørven I (1978) Ophthalmic artery pressure during retrobulbar anaesthesia. Acta Ophthalmol (Copenh) 56:574-586

6. Lende RA, Ellis PP (1964) Induced spasm in the retinal arterioles of cats I. Mechanisms and characteristics. Arch Ophthalmol 71:701-705

7. Saiki JH, Thompson S, Smith F, Atkinson R (1972) Paraplegia following intrathecal chemotherapy. Cancer 29:370-374

8. Nathan PW, Sears TA (1961) Action of methyl hydroxybenzoate on nervous conduction. Nature 192:668-669 